\title{
The Fight Against Cancer: Missiles in Nuclear Medicine
}

\section{Lutfun Nisa MBBS, MPhil and Kamila Afroj Quadir, PhD}

National Institute of Nuclear Medicine \& Allied Science

The search for the right ammunition to fight cancer has been going on for decades. Extensive research leading to development of new modern therapy proved to be partially successful in treating and prolonging the lives of patients with many common types of cancer. But an ideal therapy with high therapeutic index and negligible toxicity is yet to be devised. Hence the continuing quest for anticancer agents and procedures that can effectively select between malignant and nonmalignant cells.

A new type of therapy that has recently become popular is the proton beam therapy. The charged particles in proton therapy deliver a very high dose of radiation to the cancer but releases very little radiation to the normal tissue in their path. Thus, in theory at least, this approach minimizes damage to healthy organs and structures surrounding the cancer. While proton therapy is a giant step forward, it is yet to be the magic bullet for nonlocalized cancers like leukemia.

Going back in history, the beginning of the 20th century was significant for putting forward the concept of a magic bullet theory for cancer therapy. Paul Ehrlich the Nobel Laureate postulated an ideal therapy for disease, a toxic chemical that would pin point a biological target and act like a missile. Since that exciting concept, scientists have been searching for a magic bullet that could go straight to the source of the cancer. Eventually it took 75 years to go from Ehrlich's magic bullet theory to a laboratory created monoclonal antibody (mAb) and almost 30 years more to successfully link them to a toxic payload to target specific cancer cells. This proved to be an effective way to deliver cyrotoxic agents to the tumor. Monoclonal antibodies were thus critical to the development of immunooncology and it opened the gates for further continuous development and construction of novel targets in cancer treatment. Consequently many targeted immuno-therapeutic pharmaceuticals were developed and validated for specific tumor types. There are currently hundreds of new pathwaytargeted anticancer agents undergoing phase II and phase III clinical trials.

Targeted radionuclide therapy is just one type of therapy within the category of "targeted therapies". Monoclonal antibodies armed with radionuclides provide a means of targeting radiation therapy specifically to tumor cells that express the antigen to which the antibody was originally raised. Targeted radionuclide therapy is said to "combine the specificity of cancer cell targeting with the known antitumor effects of ionizing radiation and has the potential to simultaneously eliminate both a primary tumor site and cancer that has spread throughout the body, including malignant cell populations that are undetectable by diagnostic imaging". Targeted radionuclide therapy includes three types of particulate radiation: beta particles, alpha particles, and Auger electrons. These can radiate tissue volumes with multicellular, cellular and subcellular dimensions respectively.

With regard to beta-emitters, FDA has approval on four beta emitters: yttrium-90 and iodine-131, samarium-153-EDTMP $\quad\left(\right.$ Quadramet $\left.^{\circledR}\right)$ and strontium-89-chloride. However, the scope of preclinical and clinical research in the therapy field is much broader, involving at least eight additional beta-emitting radionuclides: lutetium-177, holmium166, rhenium-186, rhenium-188, copper-67, promethium-149, gold-199, and rhodium-105.

Targeted therapy with $\alpha$-emitting radionuclides has emerged as a particularly promising approach to molecular radiotherapy. This is evident from recent 
reviews of several preclinical studies. Compared with $\beta$-emitters such as ${ }^{90} \mathrm{Y}$ and ${ }^{131} \mathrm{I}$, radionuclides decaying by the emission of $\alpha$-particles offer several significant advantages from a radiobiological perspective. For example, the mean LET of $\alpha$ particles emitted by astatine-211 $\left({ }^{211} \mathrm{At}\right)$ is 97 $\mathrm{keV} / \mu \mathrm{m}$, a value $>400$ times higher than that for high-energy $\beta$-particles emitted by ${ }^{90} \mathrm{Y}$. The energetic $\alpha$-particles has a range of only a few cell diameters and is therefore markedly superior for destroying cancer cells and minimizing damage to distant normal tissues. In particular minimal residual or micrometastatic disease can be potentially eliminated. Research work done for more than two decades on targeted alpha therapy (TAT) has enabled the progression from in-vitro studies through in-vivo experiments and on to clinical trials. Many centers around the world, notably in Australia, the USA and Europe are using targeted alpha therapy (TAT) in selected cases. Clinical trials at these centers have demonstrated the safety, feasibility and activity of TAT in the treatment of small volume and cyto-reduced disease. However there is still the need to establish maximum tolerance doses for practical acceptance. The efficacy of TAT to reduce solid tumors by anti vascular alpha therapy is another aspect that must be studied further. Further, to initiate wide clinical acceptance of the procedure, large number of patient population must be studied. Bangladesh can take this opportunity to collaborate with other advanced centers in these clinical trials. Given the fact that both infrastructure and potential exists in the country to conduct sophisticated studies, Bangladesh would certainly benefit from the many advantages that would transpire from such collaborations.

Auger electron emitters are another group of radionuclides with potential for highly effective and very specific tumor cell killing. To kill the cancerous cells, the auger therapy relies on radiation-induced damage to cancer cells (particularly DNA damage), to arrest cell division and stop tumor growth and metastases. Auger emitters such as bromine-77, indium-111, iodine123, and iodine-125, are currently being investigated.

Conclusion: Targeted radionuclide therapy is a very exciting and rapidly growing field of nuclear medicine that holds great promise for future novel and customized approach to cancer treatment. Matrix of targeted therapeutics offering multiple radionuclide-molecular carrier combinations can provide the tools to implement targeted radionuclide therapy regimens that are optimally tailored to the needs of individual patients. The desire to move away from nonspecific toxic therapies commonly used in oncology and toward much less toxic targeted treatments, which impact only the targeted tissues is very exciting. Carrying a destructive payload directly to the disease, is the magic bullet, the missile of targeted therapy that exists within the field of Nuclear Medicine and this is without a doubt a monumental leap forward in the fight against cancer.

\section{References}

1. Allen BJ, Raja C, Rizvi S, Li Y, Tsui W, Zhang D, et al. Targeted alpha therapy for cancer. Phys Med Biol 2004; 49:3703-3712

2. Kraeber-Bodéré F, Rousseau C, Bodet-Milin C, Mathieu C, Guérard F,Frampas E et al. Tumor Immunotargeting Using Innovative Radionuclides Int. J. Mol. Sci. 2015; 1: 3932-3954

3. Michael R. Zalutsky, David A. Reardon, Oscar R. Pozzi, Ganesan Vaidyanathan, Darell D. Bigner. Targeted $\alpha$-particle radiotherapy with ${ }^{211}$ At-labeled monoclonal antibodies. Nuclear Medicine and Biology 2007; 34 (7): 779-785

4. Zalutsky MR, Vaidyanathan G. Astatine-211-labeled radiotherapeutics: an emerging approach to targeted alpha particle therapy. Curr Pharm Des 2000; 6:14331455. 